\title{
Prognosis and prognostic factors in patients with intermediate-risk papillary thyroid carcinoma defined by the Japanese clinical practice guidelines for thyroid tumours
}

\author{
Kiyomi Horiuchi* and Takahiro Okamoto \\ Department of Breast and Endocrine Surgery, Tokyo Women's Medical University, 8-1 Kawada-cho, Shinjuku-ku, Tokyo, 162-8666, Japan
}

\begin{abstract}
Objective: Japanese clinical guidelines suggest choosing an appropriate surgical strategy for papillary thyroid carcinoma (PTC) by risk classification, with lobectomy and central node dissections recommended for low-risk PTC and total thyroidectomy \pm lateral neck dissection for high-risk PTC. However, there is no specific recommendation for the intermediate-risk group.
\end{abstract}

Study design: Retrospective chart revue.

Setting: Tertiary referred centre.

Materials and Methods: 466 patients with PTC underwent surgery as an initial operation. We evaluated recurrence rate of each risk group and prognostic factors of intermediate risk group.

We used the Kaplan-Meier method to determine the recurrence rate and compared the rates using the log-rank test. To investigate the prognostic factors for patients with intermediate risk, we used the Kaplan-Meier method and the log-rank test as a univariate analysis and a Cox proportional hazards model as a multivariate analysis.

Results: We analysed data for 449 eligible patients. Overall, 10-year survival ( $95 \%$ confidence interval) was $95.0 \%$ (92.1\%-96.8\%). The 10-year cause specific survival rate in the low-, intermediate-, and high-risk groups was 100\%, 95.1\% (92.0\%-97.0\%), and 72.0\% (54.2\%-84.8\%), respectively.

Regarding the prognostic factors for patients with intermediate risk, tumour size and age and surgical massive extrathyroidal invasion beyond sterno-thyroid muscle respectively, were statistically significant for recurrence $(P=0.0016,0.0107,0.0099)$ by univariate analysis. The Cox proportional hazards odel revealed that sex, age > 55 , and tumour size $>4 \mathrm{~cm}$ were associated with recurrence $(P=0.0209,0.0189$, and 0.0091 , respectively).

Conclusion: The PTC risk classification in the Japanese clinical guidelines was very appropriate.

\section{Introduction}

Management of papillary thyroid carcinoma (PTC) is challenging because the benefits and risks of available treatments are not clearly determined for this common endocrine malignancy [1]. Total thyroidectomy followed by radioactive iodine ablation (RIA) and with thyrotropin suppression therapy has been the mainstay of the management in the United States and Europe [2], although a "risk-adapted" approach has been advocated rather than a uniform application of the interventions.

The guidelines for the management of thyroid cancer developed in Western countries served as useful references for Japanese physicinas as well, but the Japan Association of Endocrine Surgeons and the Japanese Society of Thyroid Surgery required their own guidelines for the following reasons: First, thyroid-conserving surgery, that is, less-extensive thyroidectomy, has been the most common operative procedure in Japan [3-9]. Second, the availability of RIA therapy has been quite limited because of legal restrictions and inadequate reimbursement of medical fees $[4,5,10]$. Third, adjuvant thyrotropin suppression therapy has not been adopted as a routine treatment $[4,5,10]$.

The Japanese experts classified PTC into three prognostic risk groups based on the preoperative (clinical) tumour-node-metastasis (TNM) system in their first guidelines for the management of thyroid tumours published in 2010. The groups are low-risk (T1N0M0), intermediaterisk, and high-risk (tumour size $>5 \mathrm{~cm}$, large metastatic lymph nodes $>$ $3 \mathrm{~cm}$ in diameter, extrathyroidal extension to the mucosa of the trachea or oesophagus, or distant metastasis) [6]. The experts recommended

${ }^{\star}$ Correspondence to: Kiyomi Horiuchi, Department of Breast and Endocrine Surgery, Tokyo Women's Medical University, 8-1 Kawada-cho, Shinjuku-ku, Tokyo, 162-8666, Japan. Tel: +81-03-3353-8111; E-mail: horiuchi.kiyomi@ twmu.ac.jp

Key words: Kaplan-Meier method, Cox proportional hazards model, endocrine malignancy

Received: March 13, 2020; Accepted: March 31, 2020; Published: April 03, 2020 
lobectomy with prophylactic paratracheal lymph node dissection for patients with low-risk PTC and suggested total thyroidectomy ( \pm lateral neck dissection) followed by RIA treatment and thyrotropin suppression therapy for high-risk patients. For the intermediate-risk group, the experts concluded that both lobectomy and total thyroidectomy are appropriate options as long as careful judgements are made for each patient; however, unique prognostic factors specific to this patient population were not provided in the guidelines.

This retrospective study was designed to examine the validity of the Japanese risk classification system, and to determine the clinical factors predicting oncological events in patients with intermediate-risk PTC.

\section{Patients and methods}

\section{Study population}

From the retrospective chart review, from among 454 patients who underwent their initial treatment for PTC between January 2000 and December 2004, we excluded 5 patients from the study because of a lack of relevant data (Figure 1). Each of the remaining 449 patients was classified into one of the three risk groups according to the Japanese Clinical Practice Guidelines. Distant metastasis was examined by chest $\mathrm{X}$-ray, or neck and chest computed tomography. According to the diagnostic findings, patients were classified as low-, intermediate-, or high-risk [11].

\section{Preoperative evaluations}

In addition to physical examinations, ultrasonography as well as fine needle aspiration cytology of the suspected lesions was performed for all patients. Plain chest radiography was a routine preoperative test while computed tomographic scans of the neck and chest were indicated to examine local/regional tumour extension and distant metastases in select patients. Serum levels of thyrotropin, free triiodothyronine, free thyroxine, thyroglobulin, and anti-thyroglobulin antibody were also measured.

\section{Treatment strategies}

Subtotal thyroidectomy with either prophylactic or therapeutic lymph node dissection of the central and lateral neck regions was a standard surgical procedure for patients with PTC. For some patients who underwent total thyroidectomy because of advanced-stage disease, a diagnostic whole-body scan using $5 \mathrm{mCi}$ of I-131 was performed as a postoperative evaluation. Patients with distant metastases received RIA therapy with $100 \mathrm{mCi}$ I-131. Thyrotropin suppression therapy was used only for select patients.

\section{Postoperative follow-up}

All patients were followed two or three times each year by both physical examinations and thyroid function testing, namely, thyrotropin, free triiodothyronine, free thyroxine, and thyroglobulin levels. Additionally, ultrasonography and computed tomography of the neck were used to confirm the presence or absence of cancer recurrence. Cancer recurrence was defined as both of local recurrence and distant metastasis after curative surgery.

\section{Observations}

We retrospectively reviewed patients' medical records to collect the following data for each patient: age, sex, clinical TNM stage, intraoperative findings (e.g., extrathyroidal extension, extranodal extension, macroscopic curability), pathological TNM stage, serum levels of thyrotropin and thyroglobulin at each visit, the presence or absence of local/locoregional recurrence, presence or absence of distant metastasis, and survival. The primary outcome of this study was overall survival and disease-free survival (DFS), and the secondary outcome was cause-specific survival (CSS), with the day of surgery being time zero. The third outcome was identifying the risk factors in the intermediate group.

\section{Statistical analyses}

We estimated DFS and CSS using the Kaplan-Meier method. The log-rank test was used to examine differences between the risk groups as well as to identify potential prognostic factors. Cox's proportional hazards regression modelling was used for multivariable analysis to determine the independent prognostic factors for DFS in patients with intermediate-risk PTC. The assumption of proportionality was examined using log-log plot analysis. Hazard ratios were presented with their 95\% confidence intervals (CI). All statistical tests were two-sided, and differences were considered statistically significant if $P<0.05$. All statistical analyses were performed using JMP software (version 13; SAS Institute Japan Ltd., Tokyo, Japan).

\section{Ethical considerations}

This study was approved by the Ethical Committee of Tokyo Women's Medical University (No. 4063), and the requirement to obtain informed consent was waived.

\section{Results}

\section{Patients' characteristics}

The median follow-up was 117 months (range, 0-164 months). Patients' clinical and pathological characteristics are shown in Table 1. The numbers of patients with low-, intermediate-, and high-risk PTC were $67(15 \%), 347(77 \%)$, and $35(8 \%)$, respectively. The median age was 51 years, and female patients accounted for $76 \%$ of all patients. Neither data regarding RIA examination nor serum thyrotropin suppression therapy after total thyroidectomy were obtained in this study because of a lack of precise data. Extrathyroidal extension of the tumour and extranodal extension were recognized intraoperatively in $31.6 \%$ and $1.1 \%$ of patients, respectively (Table 1 ).

\section{Oncological events}

Forty-two patients (9\%) experienced PTC recurrence as a first-time oncological event, and 11 patients (2\%) died from the disease (Table 2). Of the 33 patients who experienced recurrence in the intermediate-risk group, local recurrence, lymph node metastasis, and distant metastasis was observed in 4,26 , and 3 patients, respectively. The "recurrence" does not include the cases which had biochemical recurrence manifested by thyroglobulin only.

Overall, 5- and 10-year survival were 97.2\% (95\% CI: 95.0\%-98.4\%) and $95.0 \%$ (95\% CI: 92.1\%-96.8\%), respectively (Figure 2). Five- and 10-year DFS for the entire study population were $94.2 \%$ (95\% CI: $91 \%$ 96\%) and $89.1 \%$ (95\% CI: 85\%-92\%), respectively (Figure 3a). The $10-$ year DFS for the low-, intermediate-, and high-risk groups was $100 \%$, $89 \%$ (95\% CI: 84\%-92\%) and 74\% (95\% CI: 56\%-87\%), respectively ( $P$ $<0.001$; log-rank test; Figure 3b).

CSS values for all patients were $97.9 \%$ (95\% CI: 96\%-99\%) at 5 years and $96.6 \%$ (95\% CI: $94 \%-98 \%)$ at 10 years (Figure 4a). The 10 -year CSS for the low-, intermediate-, and high-risk groups was 100\%, 95\% (95\% CI: $92.0 \%-97.0 \%)$ and 72\% (95\% CI: 54.2\%-84.8\%), 
Horiuchi K (2020) Prognosis and prognostic factors in patients with intermediate-risk papillary thyroid carcinoma defined by the Japanese clinical practice guidelines for thyroid tumours

Table 1. Patients and characteristics

\begin{tabular}{|c|c|c|c|c|}
\hline & All & Low risk & Intermediate risk & High risk \\
\hline Number & 449 & 67 & 347 & 35 \\
\hline Male-Female & 108: 341 & $9: 58$ & $78: 269$ & $21: 14$ \\
\hline Age (years) & $51(16-86)$ & $52(22-73)$ & $51(16-86)$ & $51(18-81)$ \\
\hline Follow-up period (days) (range) & $\begin{array}{c}3532 \\
(5-4920)\end{array}$ & $\begin{array}{c}3061 \\
(5-4920)\end{array}$ & $\begin{array}{c}3637 \\
(0-4838)\end{array}$ & $\begin{array}{c}3578 \\
(28-4797)\end{array}$ \\
\hline Tumor size $(\mathrm{mm})$ & $19(0-90)$ & $11(5-20)$ & $21(0-50)$ & $40(4-90)$ \\
\hline $\mathrm{p}^{\mathrm{N}(0 / \mathrm{a} / \mathrm{lb})}$ & $118 / 114 / 216$ & $67 / 0 / 0$ & $45 / 112 / 189$ & $6 / 2 / 27$ \\
\hline $\mathrm{P}^{\mathrm{Ex} *}{ }_{(0 / 12 / \text { nodalEx } / \mathrm{X})}$ & $301 / 87 / 55 / 5 / 2$ & $67 / 0 / 0 / 0 / 0$ & $221 / 84 / 40 / 1 / 1$ & $13 / 3 / 15 / 4 / 1$ \\
\hline $\mathrm{M}(0 / 1)$ & $442 / 7$ & $67 / 0$ & $347 / 0$ & $28 / 7$ \\
\hline $\begin{array}{l}\text { Thyroid resection } \\
\text { Total/subtotal }\end{array}$ & $163 / 286$ & $12 / 55$ & $128 / 219$ & $27 / 8$ \\
\hline $\begin{array}{l}\text { Lymph node dissection** } \\
\text { Central only/lateral }\end{array}$ & $144 / 302$ & $51 / 16$ & $89 / 258$ & $4 / 31$ \\
\hline $\begin{array}{l}\text { Intraoperative findings } \\
\text { Extrathyroidal extension/Extranodal extensions }\end{array}$ & $142 / 5$ & $0 / 0$ & $124 / 1$ & $18 / 4$ \\
\hline
\end{tabular}

*Ex: Extra thyroidal extension.

Table 2. Recurrence and death rates

\begin{tabular}{|l|c|c|c|c|}
\hline & Low risk & $\begin{array}{c}\text { Intermediate } \\
\text { risk }\end{array}$ & High risk & Total \\
\hline Number of patients & 67 & 347 & 35 & \\
\hline Recurrence & & & & $42(9 \%)$ \\
\hline Local recurrence & $0(0 \%)$ & $4(1 \%)$ & $2(7 \%)^{*}$ & \\
\hline Lymph node metastasis & $0(0 \%)$ & $26(7 \%)$ & $5(18 \%)^{*}$ & \\
\hline Distant metastasis & $0(0 \%)$ & $3(1 \%)$ & $2(6 \%)^{*}$ & \\
\hline Death from PTC** & $0(0 \%)$ & $4(1 \%)$ & $7(20 \%)$ & $11(2 \%)$ \\
\hline
\end{tabular}

*Recurrence rate was calculated in 28 patients with MO. **PTC: Papillary Thyroid Carcinoma.

Table 3. Cox proportional hazards model in the intermediate-risk group

\begin{tabular}{|l|c|c|c|}
\hline Factors & Hazard ratio & $\begin{array}{c}\mathbf{9 5 \%} \text { Confidence } \\
\text { internal }\end{array}$ & P-value \\
\hline Male & 2.41 & $1.14-4.90$ & $0.0209^{*}$ \\
\hline Age $>55$ years & 2.33 & $1.15-4.89$ & $0.0189^{*}$ \\
\hline $\begin{array}{l}\text { Tumour diameter } \\
>4 \mathrm{~cm}\end{array}$ & 3.14 & $1.35-6.67$ & $0.0091^{*}$ \\
\hline $\begin{array}{l}\text { Extra thyroidal or extra nodal } \\
\text { extension }\end{array}$ & 1.72 & $0.84-3.51$ & 0.1295 \\
\hline
\end{tabular}

* Statistically significant.

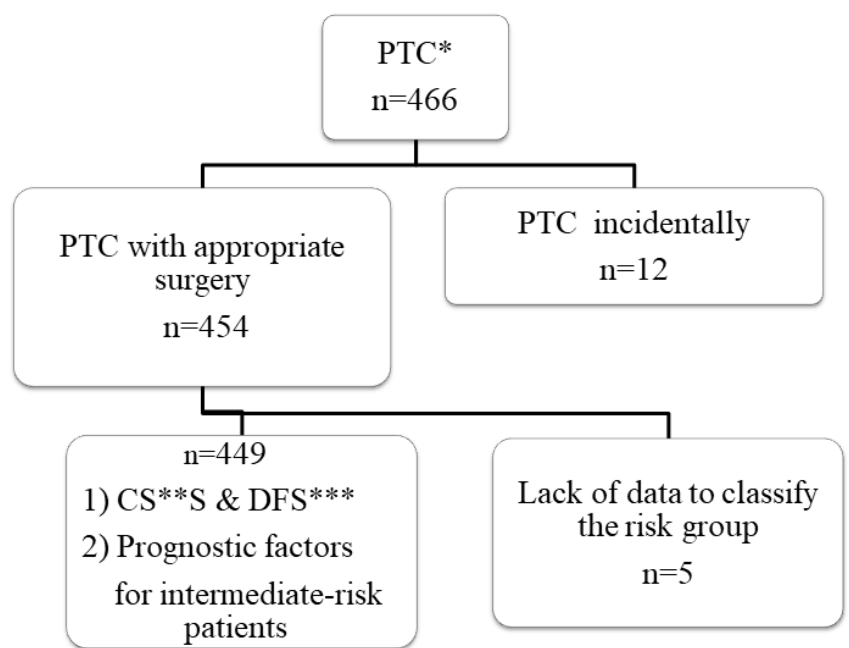

*PTC: papillary thyroid carcinoma, ${ }^{* *} \mathrm{CSS}$ : cause specific survival

***DFS : disease free survival

Figure 1. Patient selection algorithm respectively (Figure 4b). There was no difference between the low- and intermediate-risk groups; however, high-risk patients had significantly worse CSS compared with the other two groups $(P<0.001)$.

\section{Prognostic factors for DFS in patients with intermediate-risk PTC}

The Kaplan-Meier curves for DFS in patients with intermediaterisk PTC indicated that male sex, age $>55$ years, tumour size $>4 \mathrm{~cm}$, and extrathyroidal extension were associated with worse prognosis while clinically node-positive status was not prognostic (Fig. 5). A Cox's proportional hazards model with the four variables significant in the univariate analyses revealed that male sex (hazard ratio: 2.41, 95\% CI: 1.14-4.90), tumour size $>4 \mathrm{~cm}$ (hazard ratio: $3.14,95 \% \mathrm{CI}$ : $1.35-6.67$ ), and age $>55$ years (hazard ratio: 2.33 , 95\% CI: $1.15-4.89$ ) were significant independent factors predictive of DFS (Table 3 ).

\section{Discussion and conclusion}

This was a retrospective study of the validity of the risk classification in the Japanese clinical guidelines for PTC. Overall survival and CSS in the three risk groups differed significantly, especially when comparing the low- and intermediate-risk groups with the high-risk group. Our results revealed that the surgical strategy for low- and high-risk groups suggested in the Japanese clinical guidelines were valid. Our results showed controversy of benefit with total thyroidectomy followed RIA therapy for patients with low-risk PTC according to the excellent prognosis in this group, even without RIA treatment. In contrast, total thyroidectomy + lateral neck dissection followed by RIA might be mandatory for high-risk PTC. Because our results revealed that DFS and CSS in both the low- and intermediate-risk groups were high, postoperative radioiodine ablation might be controversial for the patients with an intermediate-risk.

An important part of our study was our attempt to determine the prognostic factors in the intermediate-risk group because this analysis has not been performed previously. Our results indicated a small benefit of postoperative RIA in patients with intermediate-risk PTC because they have an excellent prognosis, similar to the low-risk group. Even though only $57 \%$ of patients underwent total thyroidectomy followed by only $10 \mathrm{mCi}$ iodine for RIA in the intermediate-risk group, 5- and 10 -year DFS were very high at $99 \%$ and $88.9 \%$, respectively. Narrowing the CIs made these results reliable.

The Japanese surgical strategy for PTC differs from that in Western countries [3-5]. In particular, excellent results have been achieved for low-risk PTC even with hemithyroidectomy [6-8]. 


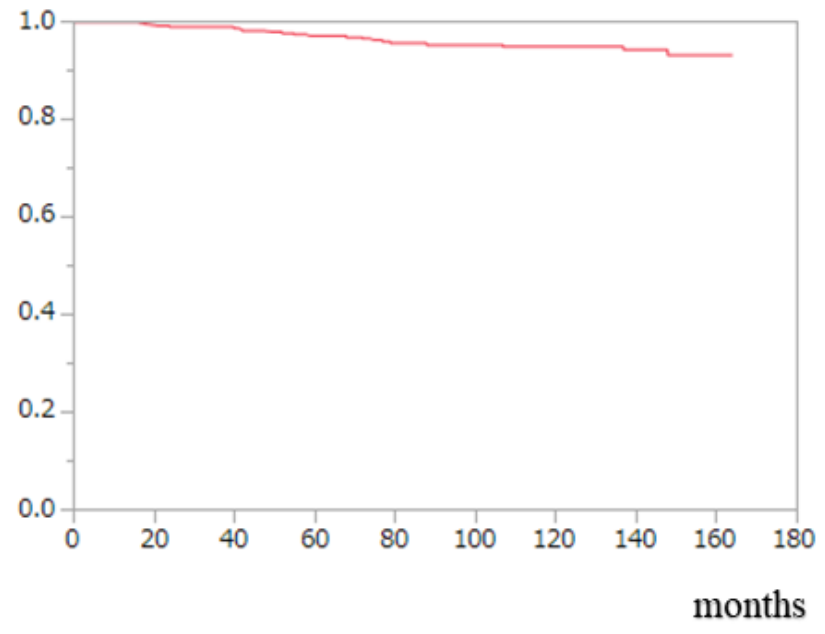

\begin{tabular}{lll} 
& $\begin{array}{l}\text { 5-year } \\
\left(95 \% \mathrm{CI}^{*}\right)\end{array}$ & $\begin{array}{l}\text { 10-year } \\
(95 \% \mathrm{CI})\end{array}$ \\
\hline Survival (\%) & 97.2 & 95.0 \\
& $(95.0-98.4)$ & $(92.1-96.8)$
\end{tabular}

${ }^{*} \mathrm{CI}$ : confidence interval

Figure 2. Overall survival

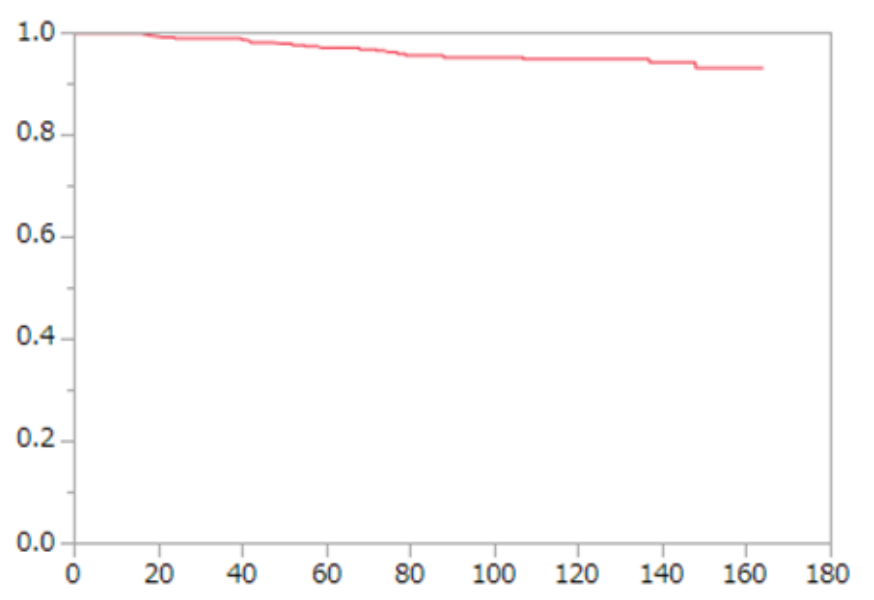

months

\begin{tabular}{lll} 
& $\begin{array}{l}\text { 5-year } \\
\left(95 \% \mathrm{CI}^{*}\right)\end{array}$ & $\begin{array}{l}10-\text { year } \\
(95 \% \mathrm{CI})\end{array}$ \\
\hline Survival (\%) & $\begin{array}{l}97.2 \\
(95.0-98.4)\end{array}$ & $\begin{array}{l}95.0 \\
\end{array}$ \\
& &
\end{tabular}

${ }^{*} \mathrm{CI}$ : confidence interval

Figure 3. Disease-free survival a) Overall

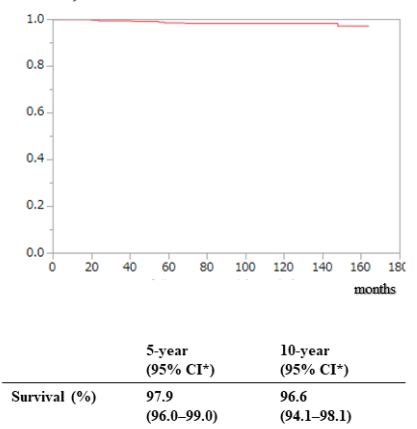

${ }^{*} \mathrm{CI}$ : confidence interval b) By risk group

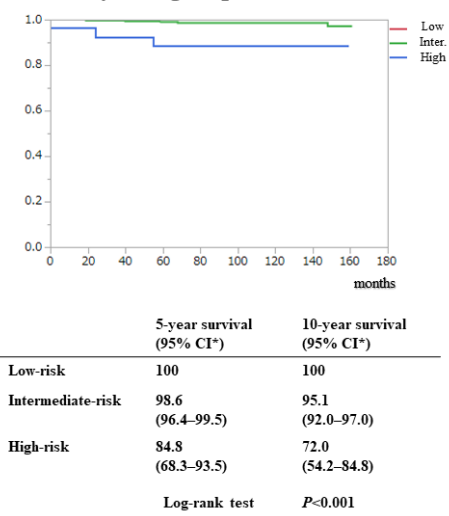

Log-rank test $\quad P<0.001$
Figure 4. Cause-specific survival

Regarding postoperative thyrotropin suppression therapy, Sugitani et al. [10], reported no benefit for low-risk PTC. Given these findings, Japanese surgeons have attempted to perform risk-adapted surgery and postoperative treatment.

Several studies have reported the risk factors for differentiated thyroid cancer or PTC. Matsuzu et al. [7], reported that risk factors related to distant metastasis were age $>45$ years, tumour size $>4$ $\mathrm{cm}$, extrathyroidal invasion positive, and lymph node-positive [7]. Ito et al. [12] reported that the risk factors related to DFS for lymph node metastasis were age $>55$ years, male sex, massive extrathyroidal extension, and tumour size $>3 \mathrm{~cm}$ [13]. Additionally, Barney et al. [14], revealed that factors related to CSS were tumour size $>4 \mathrm{~cm}$ and age $>45$ years. Although there has been interest in the prognostic factors in PTC, we could not compare these studies' results with our results because most studies included combined data for the low- and intermediate-risk groups. No reports analysed the prognostic factors exclusively for patients with intermediate risk. To our knowledge, ours is the first study to analyse the prognostic factors in patients with intermediate-risk PTC, even though this was a retrospective study. Our results revealed that the Japanese risk classification of PTC was very accurate regarding determining prognosis, and there was no evidence against the surgical strategy for low- and high-risk PTC in the Japanese guidelines. Our aim in this study was to evaluate the prognosis of the intermediate-risk group because no previous study has done so. Our results indicated that the prognostic factors in patients with intermediate-risk PTC were 1) male sex, 2) tumour size $>4 \mathrm{~cm}$, and 3) age $>55$ years. Interestingly, the prognostic factors of age $>55$ years and tumour size $>4 \mathrm{~cm}$ in diameter were the same as results reported in the $8^{\text {th }}$ TNM classification [15]. Furthermore, although we did not analyse the efficacy of postoperative RIA for intermediate-risk patients, it is crucial to know if postoperative RIA is mandatory in this risk group. Several reports have evaluated the efficacy of ablation therapy after total thyroidectomy for differentiated thyroid cancer [16], and some reports concluded that ablation prolongs survival in these patients $[17,18]$. Lamartina et al. [19], performed a systematic review of the literature evaluating RIA for low-risk differentiated thyroid cancer and concluded that there was no evidence that RIA decreased the recurrence rate in patients with low- or intermediate-risk thyroid cancer. Schvartz et al. [20], also failed to prove any survival benefit of RIA for low-risk PTC.

There are limitations in our study. First, this was a retrospective study, and the surgical strategy at the time of operation differed from 
Sex

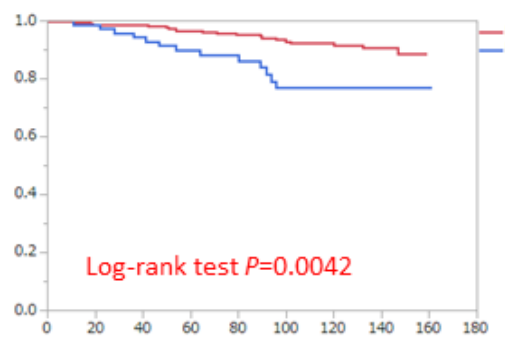

days
Age $>55$ years

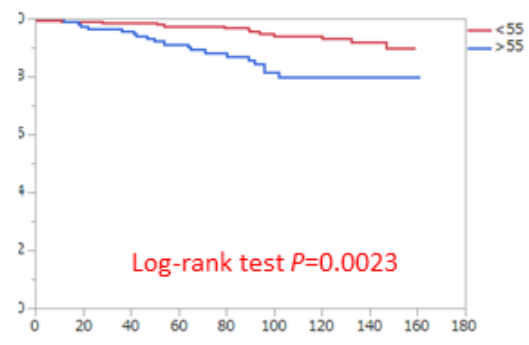

days

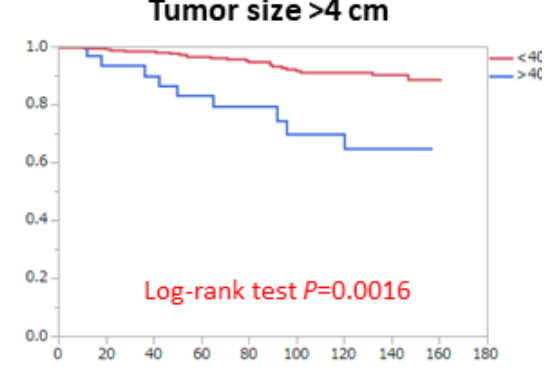

days

Lymph node metastasis

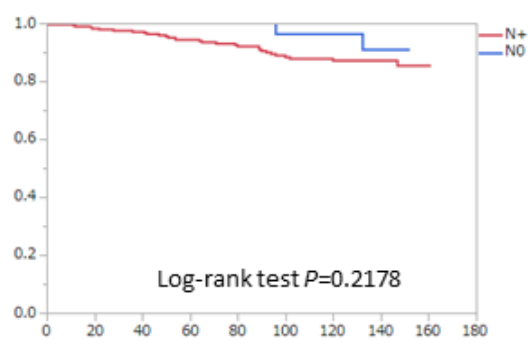

days
Extrathyroidal invasion

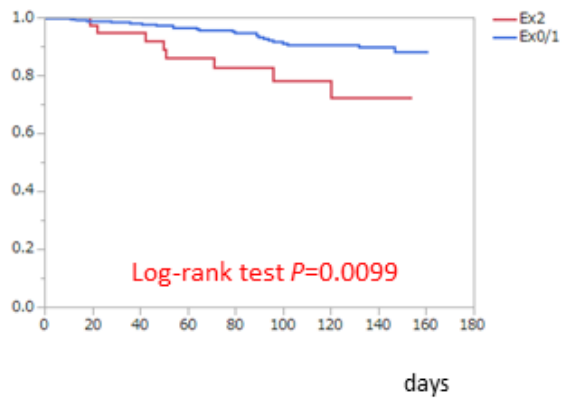

Figure 5. Prognostic factors for disease-free survival in the intermediate-risk group

the guidelines. Subtotal thyroidectomy was a standard surgical strategy for PTC and was performed most often in the high-risk group in 1990's. Second, we classified the risk groups according to histopathological diagnosis. Patients should have been classified according to preoperative information, but there was insufficient preoperative information to do so. Third, the intermediate group in the Japanese classification system differs from that of the American Thyroid Association [2]. Basically, the Japanese risk classification is determined according to preoperative information as for decision making regarding the surgical strategy (lobectomy or thyroidectomy), but the American Thyroid Association's classification requires pathological information and demonstrates continuum of risk the low and intermediate groups.

We have a future perspective. In December 2018, the Japanese Association of Endocrine Surgery announced revised guidelines, and we plan to analyse our data according to the new guidelines, in the near future.

\section{References}

1. Seib CD, Sosa JA (2019) Evolving Understanding of the Epidemiology of Thyroid Cancer. Endocrinol Metab Clin North Am 48: 23-35. [Crossref]

2. Haugen BR, Alexander EK, Bible KC, Doherty GM, Mandel SJ, et al. (2016) 2015 American Thyroid Association Management Guidelines for Adult Patients with Thyroid Nodules and Differentiated Thyroid Cancer: The American Thyroid Association Guidelines Task Force on Thyroid Nodules and Differentiated Thyroid Cancer. Thyroid 26: 1-133. [Crossref]

3. Noguchi S, Murakami N, Kawamoto H (1994) Classification of papillary cancer of the thyroid based on prognosis. World J Surg 18: 552-557; discussion 558. [Crossref]

4. Shigematsu N, Takami H, Ito N, Kubo A (2005) Nationwide survey on the treatment policy for well-differentiated thyroid cancer -- results of a questionnaire distributed at the 37th meeting of the Japanese Society of Thyroid Surgery. Endocr J 52: 479-491. [Crossref]

5. Shigematsu N, Takami H, Kubo A (2006) Unique treatment policy for welldifferentiated thyroid cancer in Japan: results of a questionnaire distributed to members of the Japanese Society of Thyroid Surgery and the International Association of Endocrine Surgeons. Endocr J 53: 829-839. [Crossref]
6. Ito Y, Masuoka H, Fukushima M, Inoue H, Kihara M, et al. (2010) Excellent prognosis of patients with solitary T1N0M0 papillary thyroid carcinoma who underwent thyroidectomy and elective lymph node dissection without radioiodine therapy. World J Surg 34: 1285-1290. [Crossref]

7. Matsuzu K, Sugino K, Masudo K, Nagahama M, Kitagawa W, et al. (2014) Thyroid lobectomy for papillary thyroid cancer: long-term follow-up study of 1,088 cases. World J Surg 38: 68-79. [Crossref]

8. Ebina A, Sugitani I, Fujimoto Y, Yamada K (2014) Risk-adapted management of papillary thyroid carcinoma according to our own risk group classification system: is thyroid lobectomy the treatment of choice for low-risk patients? Surgery 156: 15791588; discussion 1588-1579.

9. Dong W, Horiuchi K, Tokumitsu H, Sakamoto A, Noguchi E, et al. (2019) TimeVarying Pattern of Mortality and Recurrence from Papillary Thyroid Cancer: Lessons from a Long-Term Follow-Up. Thyroid 29: 802-808. [Crossref]

10. Sugitani I, Fujimoto Y (2010) Does postoperative thyrotropin suppression therapy truly decrease recurrence in papillary thyroid carcinoma? A randomized controlled trial. $J$ Clin Endocrinol Metab 95: 4576-4583. [Crossref]

11. Takami H, Ito Y, Okamoto T, Yoshida A (2011) Therapeutic Strategy for Differentiated Thyroid Carcinoma in Japan Based on a Newly Established Guideline Managed by Japanese Society of Thyroid Surgeons and Japanese Association of Endocrine Surgeons. World J Surg 35: 111-121. [Crossref]

12. Ito Y, Miyauchi A, Jikuzono T, Higashiyama T, Takamura Y, et al. (2017) Risk factors contributing to a poor prognosis of papillary thyroid carcinoma: validity of UICC/ AJCC TNM classification and stage grouping. World J Surg 31: 838-848. [Crossref]

13. Ito Y, Hirokawa M, Jikuzono T, Higashiyama T, Takamura Y, et al. (2007) Extranodal tumor extension to adjacent organs predicts a worse cause-specific survival in patients with papillary thyroid carcinoma. World J Surg 31: 1194-1201. [Crossref]

14. Barney BM, Hitchcock YJ, Sharma P, Shrieve DC, Tward JD (2011) Overall and causespecific survival for patients undergoing lobectomy, near-total, or total thyroidectomy for differentiated thyroid cancer. Head Neck 33: 645-649. [Crossref]

15. Tuttle RM, Haugen B, Perrier ND (2017) Updated American Joint Committee on Cancer/ Tumor-Node-Metastasis Staging System for Differentiated and Anaplastic Thyroid Cancer (Eighth Edition): What Changed and Why? Thyroid 27: 751-756. [Crossref]

16. Higashi T, Nishii R, Yamada S, Nakamoto Y, Ishizu K, et al. (2011) Delayed initial radioactive iodine therapy resulted in poor survival in patients with metastatic differentiated thyroid carcinoma: a retrospective statistical analysis of 198 cases. $\mathrm{J} \mathrm{Nucl}$ Med 52: 683-689. [Crossref] 
Horiuchi K (2020) Prognosis and prognostic factors in patients with intermediate-risk papillary thyroid carcinoma defined by the Japanese clinical practice guidelines for thyroid tumours

17. Sawka AM, Thephamongkhol K, Brouwers M, Thabane L, Browman G, et al. (2004) Clinical review 170: A systematic review and metaanalysis of the effectiveness of radioactive iodine remnant ablation for well-differentiated thyroid cancer. $J$ Clin Endocrinol Metab 89: 3668-3676. [Crossref]

18. Ruel E, Thomas S, Dinan M, Perkins JM, Roman SA, et al. (2015) Adjuvant radioactive iodine therapy is associated with improved survival for patients with intermediate-risk papillary thyroid cancer. J Clin Endocrinol Metab 100: 1529-1536. [Crossref]
19. Lamartina L, Durante C, Filetti S, Cooper DS (2015) Low-risk differentiated thyroid cancer and radioiodine remnant ablation: a systematic review of the literature. $J$ Clin Endocrinol Metab 100: 1748-1761. [Crossref]

20. Schvartz C, Bonnetain F, Dabakuyo S, Gauthier M, Cueff A, et al. (2012) Impact on overall survival of radioactive iodine in low-risk differentiated thyroid cancer patients. J Clin Endocrinol Metab 97: 1526-1535. [Crossref]

Copyright: $\odot 2020$ Horiuchi K. This is an open-access article distributed under the terms of the Creative Commons Attribution License, which permits unrestricted use, distribution, and reproduction in any medium, provided the original author and source are credited. 